\title{
Effects of a combination of puerarin, baicalin and berberine on the expression of proliferator-activated receptor- $\gamma$ and insulin receptor in a rat model of nonalcoholic fatty liver disease
}

\author{
WEIHAN ZHAO*, LIJUAN LIU*, YUNLIANG WANG, TANGYOU MAO and JUNXIANG LI \\ Gastroenterology Department, Dongfang Hospital, Beijing University of Chinese Medicine, Beijing 100078, P.R. China
}

Received January 11, 2015; Accepted September 25, 2015

DOI: $10.3892 /$ etm.2015.2846

\begin{abstract}
Nonalcoholic fatty liver disease (NAFLD) is a prevalent disease, with a clinical spectrum ranging from simple fatty liver disease to nonalcoholic steatohepatitis and cirrhosis. Puerarin, baicalin and berberine are herbal products widely used in Asia, which are believed to possess therapeutic benefits for alleviating the symptoms of NAFLD. In the present study, a rat model of NAFLD, induced by a high-fat diet, was established and orthographical experimentation was used to investigate the effects of various combinations of puerarin, baicalin and berberine on the hepatic expression of proliferator-activated receptor (PPAR) $-\gamma$ and insulin receptor (IR). The present study demonstrated that serum levels of total cholesterol, alanine transaminase and low-density lipoproteins were significantly decreased in the puerarin-dominated groups $(\mathrm{P}<0.05$ vs. the model group), whereas the concentrations of tumor necrosis factor- $\alpha$ and interleukin- 6 were significantly improved in the baicalin- and berberine-dominated groups $(\mathrm{P}<0.05$ vs. the model group). Furthermore, as compared with the control group, the levels of PPAR- $\gamma /$ IR mRNA and protein expression were significantly decreased in the model group $(\mathrm{P}<0.01)$, and significantly increased in the rosiglitazone group and some of the orthogonal experiment groups $(\mathrm{P}<0.01)$. In conclusion, a combination of puerarin, baicalin and berberine induced favorable effects on NAFLD by upregulating hepatic PPAR $-\gamma$ and IR expression levels, and different proportions of monomer compositions exerted variable positive effects on various stages of NAFLD.
\end{abstract}

Correspondence to: Professor Junxiang Li, Gastroenterology Department, Dongfang Hospital, Beijing University of Chinese Medicine, 6 Fangxingyuan 1 Qu, Beijing 100078, P.R. China E-mail:weihanzhaodoc@163.com

*Contributed equally

Key words: puerarin, baicalin, berberine, non-alcoholic fatty liver disease, proliferator-activated receptor- $\gamma$, insulin receptor

\section{Introduction}

Nonalcoholic fatty liver disease (NAFLD) is characterized by an accumulation of lipids within hepatocytes, and is considered the hepatic manifestation of the metabolic syndrome (1). NAFLD develops progressively from simple steatosis to nonalcoholic steatohepatitis (NASH), fibrosis and cirrhosis (2,3). Previous studies have suggested a 'two-hit' theory for the pathogenesis of NAFLD, including insulin resistance, oxidative stress, lipid peroxidation and mitochondrial dysfunction (4,5), with insulin resistance considered the major factor.

Proliferator-activated receptor (PPAR) $-\gamma$ is a member of the nuclear hormone receptor family and as such is activated by its ligand (6). Previous studies have demonstrated that this transcription factor is associated with insulin resistance, lipid metabolism and the regulation of inflammation in fatty liver disease (7-9). The insulin receptor (IR) is a well-studied receptor tyrosine kinase, which is associated with the regulation of peripheral glucose metabolism $(10,11)$; therefore, a defect in IR may directly affect the role of insulin in the body, leading to insulin resistance and abnormal glucose tolerance (12). Previous studies have demonstrated that PPAR- $\gamma$ and IR are closely associated with insulin resistance $(13,14)$, and PPAR- $\gamma$ agonists, such as thiazolidinediones (TZDs), are able to increase insulin sensitivity in adipose, liver and skeletal muscle tissue (15). However, TZDs possess numerous side effects, including significant weight gain and peripheral edema $(16,17)$; therefore, it is necessary that novel agents that target PPAR- $\gamma$ with reduced adverse effects be developed.

Previous studies have revealed the therapeutic effects of integrative medicine in treating patients with metabolic syndrome $(18,19)$. Puerarin, baicalin and berberine (Fig. 1A-C), are three major constituents of the Chinese herbs: Puerariae Radix [roots of Pueraria lobata (Willd.) Ohwi, PR], Scutellariae Radix (roots of Scutellaria baicalensis Georgi SR) and Coptidis Rhizoma (rhizomes of Coptis chinensis Franch, CR), which have demonstrated therapeutic effects on rats models of NAFLD (20-22). It is believed that a combination of agents may effectively reduce side effects and improve adaptive resistance. In the present study, an orthogonal experimental design was used to investigate the various effects of puerarin, baicalin and berberine on rats with high-fat diet-induced NAFLD. The present 
study aimed to elucidate the complex interactions of this multicomponent disease, and further clarify the potential for natural combination pharmacological therapies for patients with NAFLD.

\section{Materials and methods}

Experimental animals and modeling. A total of 96 adult male Sprague-Dawley rats weighing 150-180 g were obtained from the Animal Breeding Center of the Beijing Vital River Laboratories Company (Beijing, China). The rats were individually housed at $22 \pm 2^{\circ} \mathrm{C}$ with a relative humidity of $50 \pm 10 \%$ and a $12 \mathrm{~h}$ light/dark cycle. After 7 days of adaptive feeding, the rats were randomly divided into control $(n=8)$, model $(n=8)$, rosiglitazone treatment $(\mathrm{n}=8)$, and nine orthogonal experiment groups $(\mathrm{n}=8 \times 9)$. The control group was fed a normal laboratory diet with free drinking water, whereas the model group, rosiglitazone treatment group $(\mathrm{R})$ and orthogonal experiment groups (A-I) were fed a normal diet supplemented with $2 \%$ cholesterol and $10 \%$ lard, for 8 weeks. This study was approved by the Review Board of the Beijing University of Chinese Medicine (Beijing, China). All animal studies were conductedc in accordance with the regulations and guidelines for the use and care of experimental animals of the Beijing University of Chinese Medicine (Beijing, China).

Therapeutic agents and reagents. Puerarin, baicalin, and berberine (Fig. 1) were purchased from Nanjing Zelang Medical Technology Co., Ltd. (Jiangsu, China). Rosiglitazone was purchased from Chengdu Hengrui Biotech Co., Ltd (Chengdu, China). Colorimetric kits for testing triglyceride (TG), total cholesterol (TC), high-density lipoprotein (HDL), low-density lipoprotein (LDL), alanine aminotransferase (ALT) and aspartate aminotransferase (AST) were purchased from BioSino Biotechnology and Science, Inc. (Beijing, China). ELISA kits for tumor necrosis factor (TNF)- $\alpha$ and interleukin (IL)- 6 were purchased from Shanghai Yanji Biotechnology Co., Ltd. (Shanghai, China). Rabbit anti-PPAR $\gamma$ (ab27649, 1:2,000), mouse anti-IR (cat. no. ab69508, 1:5,000) and goat anti-GAPDH (ab9483, 1:20,000) antibodies were purchased from Abcam (Cambridge, UK). Additional reagents were obtained from Sigma-Aldrich (St. Louis, MO, USA).

Orthogonal experimental design and intervention. Based on single-factor experimental results, three independent parameters: Puerarin, baicalin and berberine doses $(\mathrm{mg})$, were confirmed as the major influencing factors (23). A L9(34) orthogonal experimental design was conducted to evaluate the effects of the independent variables on the treatment of rats with NAFLD. The levels and ranges of the individual variables are outlined in Table I. The experiment design is presented in Table II, along with the experimental data. All experiments were performed in duplicate.

Measurement of serum biochemical parameters. Blood samples from the rats were obtained via abdominal aortic puncture and centrifuged at $644 \mathrm{x} \mathrm{g}$ for $10 \mathrm{~min}$, in order to isolate the serum. Serum ALT, AST, TC, TG, LDL, and HDL concentrations were determined using a Vitros 250 automatic biochemical analyzer (Johnson \& Johnson, Rochester, NY, USA).
Table I. Factors and levels of orthogonal experimental design.

\begin{tabular}{lccc}
\hline Levels & Puerarin $(\mathrm{mg})$ & Baicalin $(\mathrm{mg})$ & Berberine $(\mathrm{mg})$ \\
\hline 1 & 200 & 100 & 100 \\
2 & 100 & 50 & 50 \\
3 & 50 & 25 & 25 \\
\hline
\end{tabular}

Table II. Orthogonal experimental design.

\begin{tabular}{lccc}
\hline Test & Puerarin & Baicalin & Berberine \\
\hline A & 1 & 1 & 1 \\
B & 1 & 2 & 2 \\
C & 1 & 3 & 3 \\
D & 2 & 1 & 2 \\
E & 2 & 2 & 3 \\
F & 2 & 3 & 1 \\
G & 3 & 1 & 3 \\
H & 3 & 2 & 1 \\
I & 3 & 3 & 2 \\
\hline
\end{tabular}

A<smiles>O=c1c(-c2ccc(O)cc2)coc2c([C@@H]3O[C@H](CO)[C@@H](O)[C@H](O)[C@H]3O)c(O)ccc12</smiles>

B<smiles>O=C(O)C1OC2OC(Oc3cc4oc(-c5ccccc5)cc(=O)c4c(O)c3O)C(O)C1O2</smiles>

C<smiles>COc1ccc2c(c1OC)CN1CCc3cc4c(cc3C1C2)OCO4</smiles>

Figure 1. Chemical structures of (A) puerarin, $(\mathrm{B})$ baicalin, and $(\mathrm{C})$ berberine.

Histological examination. All rats survived to the final analysis and rats were sacrificed by an intraperitoneal injection 
of $10 \%$ chloral hydrate at the $8^{\text {th }}$ week. In accordance with the frozen section technique, one fresh section of liver tissue from each rat was maintained in liquid nitrogen for 4-10 sec prior to staining with Oil Red O (Sigma-Aldrich). An additional liver tissue section was fixed by immersion in $10 \%$ buffered formalin for paraffin embedding, prior to staining with hematoxylin and eosin (Beijing CellChip Biotechnology Co. Ltd., Beijing, China). The samples were examined using an Olympus CK40 inverted microscope (Olympus, Tokyo, Japan).

Detection of TNF- $\alpha$ and IL- 6 in the serum and liver tissue. The concentrations of TNF- $\alpha$ and IL- 6 in the serum and liver tissue of the rats were analyzed using commercially available ELISA kits, according to the manufacturer's instructions. Briefly, the tissue was homogenized in an ice bath with ultrasonic irradiation. After $30 \mathrm{~min}$, the homogenate was centrifuged for $15 \mathrm{~min}$ at $15,000 \mathrm{x} \mathrm{g}$ at a temperature of $4^{\circ} \mathrm{C}$. The supernatant was extracted in order to determine the concentration of protein. All samples were diluted to 1:10 and the absorbance was read at $450 \mathrm{~nm}$ using a microplate reader (Multiskan MK3; Thermo Fisher Scientific Inc., Rockford, IL, USA). Samples and standards were performed in triplicate.

Detection of the mRNA expression levels of PPAR- $\gamma$ and $I R$ by reverse transcription-quantitative polymerase chain reaction $(R T-q P C R)$. Total RNA was isolated from the liver samples using TRIzol ${ }^{\circledR}$ reagent (Invitrogen Life Technologies, Carlsbad, CA, USA) and subsequently reverse-transcribed at $42^{\circ} \mathrm{C}$ for 60 min using the oligodT primers and Super Script RT (Invitrogen Life Technologies). The primer sequences were as follows: PPAR- $\gamma$, forward 5'-TACCAC GGTTGATTTCTC-3', reverse 5'-GCTCTACTTTGATCG CACT-3'; IR, forward 5'-CTAAGGCAGATGACATCGTT-3', reverse 5'-GCTCCTCATCACCATATCGC-3'; GAPDH, forward 5'-TGGAGTCTACTGGCGTCTT-3', reverse 5'-TGT CATATTTCTCGTGGTTCA-3'. All primers were synthesized by Shanghai Yingjun Biotechnology Company (Shanghai, China). Following cDNA synthesis, $3 \mu \mathrm{l}$ cDNA was used in a 50- $\mu$ l quantitative RT-qPCR reaction including $1 \mathrm{X} \mathrm{SYBR}^{\circledR}$ Green Mix (Toyobo, Osaka, Japan) and 7.5 pmol of each specific primer. qPCR was conducted using a 7900HT Fast Real-Time PCR system (Applied Biosystems, CA, USA) with the following cycle conditions: $50^{\circ} \mathrm{C}$ for $2 \mathrm{~min}, 95^{\circ} \mathrm{C}$ for $10 \mathrm{~min}$ and then 40 cycles of $95^{\circ} \mathrm{C}$ for $5 \mathrm{~min}, 94^{\circ} \mathrm{C}$ for $15 \mathrm{sec}, 60^{\circ} \mathrm{C}$ for $30 \mathrm{sec}, 72^{\circ} \mathrm{C}$ for $30 \mathrm{sec}$ and a final extension of $72^{\circ} \mathrm{C}$ for $5 \mathrm{~min}$. The PCR products with the highest content were diluted by 1:1, 1:10, 1:100, 1:1,000, 1:10,000. PPAR- $\gamma /$ IR mRNA expression levels were calculated using the ${ }^{\Delta \Delta} \mathrm{Ct}$ method (24) using Sequence Detection system 2.1 software (Eppendorf Co., Ltd., Hamburg, Germany). The relative PPAR- $\gamma /$ IR mRNA expression levels were normalized to the expression of GAPDH in the samples. PCR amplifications were simultaneously performed in duplicate to avoid systematic errors.

Western blot analysis for the detection of PPAR- $\gamma$ and IR protein expression. Proteins in the liver tissue homogenates were extracted using ice-cold tissue lysis buffer. Protein concentrations were determined using a bicinchoninic acid (BCA) protein assay kit (Promega, Madison,WI, USA). The liver tissue samples were homogenized in radioimmunoprecipitation assay lysis buffer (BioTeke, Co. Ltd., Beijing, China) containing a phenylmethylsulfonyl fluoride protease inhibitor (BioTeke, Co. Ltd.), and the total protein concentrations were subsequently determined using a BCA protein assay. Proteins $(\sim 50 \mu \mathrm{g})$ were loaded onto 5-10\% SDS-PAGE gels and transferred to a polyvinylidene difluoride membrane (Bio-Rad Laboratories Inc., Hercules, CA, USA) for $70 \mathrm{~min}$ at $100 \mathrm{~V}$. The membranes were then incubated in blocking buffer for $2 \mathrm{~h}$ prior to the addition of primary antibodies, including PPAR $-\gamma$ and IR, which were then incubated at $4{ }^{\circ} \mathrm{C}$ overnight. The membranes were immunoblotted with primary antibodies that recognized PPAR $\gamma$ (dilution, 1:2,000), IR (dilution, 1:5,000) and GAPDH (dilution, 1:5,000). The secondary antibodies [goat anti-rabbit immunoglobulin G (IgG; cat.no. 554020) and goat anti mouse IgG (cat.no. 551011) secondary antibodies; BD Biosciences, Franklin Lakes, NJ, USA)] were diluted to 1:1,000 and incubated at room temperature for $2 \mathrm{~h}$. Proteins were detected via enhanced chemiluminescence, and the intensity of protein bands was quantified using ImageJ version 1.37 software (National Institutes of Health, Bethesda, MD, USA).

Statistical analysis. All data were analyzed using SPSS software version 17.0 (SPSS, Inc., Chicago, IL, USA). Comparisons between the groups were analyzed for statistical significance using one-way analysis of variance and Wilcoxon's signed-rank test. $\mathrm{P}<0.05$ was considered to indicate a statistically significant difference.

\section{Results}

Histological changes in the liver. Typical steatosis, balloon degeneration in hepatocytes and evident infiltration by inflammatory cells in the intercellular substance, were observed in the model group liver tissue samples (Fig. 2K), as compared with that of the control group (Fig.2L). However, these changes in pathology were alleviated in the orthogonal experiment and rosiglitazone treatment groups (Figs. 2A-I and J, respectively). Furthermore, Oil Red O staining was used to detect the quantities of lipids in the hepatocytes, the results of which demonstrated that hepatocyte lipidosis was significantly increased in the model group (Fig. 3K); however, the deposition of lipid droplets in the hepatocytes was markedly reduced in the orthogonal experiment groups and rosiglitazone treatment group (Figs. 3A-I and J, respectively).

\section{Effects of puerarin, baicalin and berberine on the biochemical parameters}

Plasma HDL, LDL, TG and TC levels. Compared with the control group, the serum HDL levels in the model group were significantly decreased $(\mathrm{P}<0.01)$; whereas the levels of LDL, $\mathrm{TG}$, and $\mathrm{TC}$ were significantly increased $(\mathrm{P}<0.01)$. However, no significant differences in the HDL levels of rats were determined between the orthogonal experiment groups $(\mathrm{P}>0.05$, Fig. 4C), and the model group. The levels of TG in groups A, D, F, G and R were significantly decreased ( $\mathrm{P}<0.05$, Fig. 4A), as were the TC levels in groups A-C and $\mathrm{E}(\mathrm{P}<0.05$, Fig. $4 \mathrm{~B})$, and the levels of LDL in groups A-D and R ( $<<0.05$, Fig. 4D), as compared with the model group. 
A

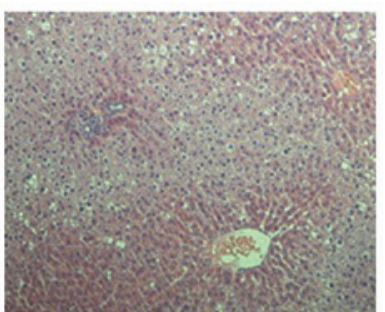

$\mathbf{E}$

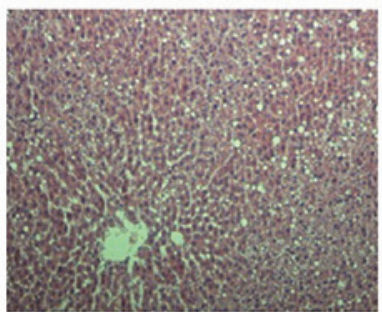

I

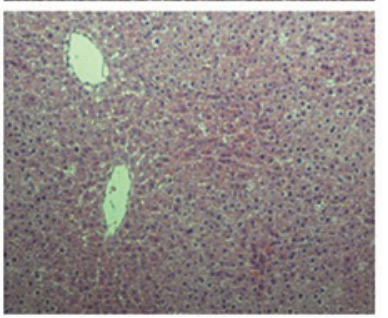

B

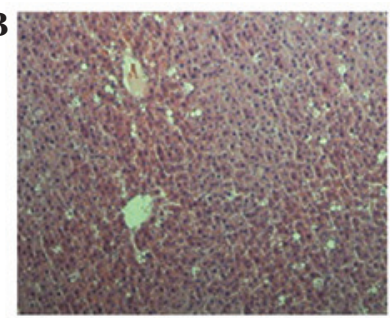

F
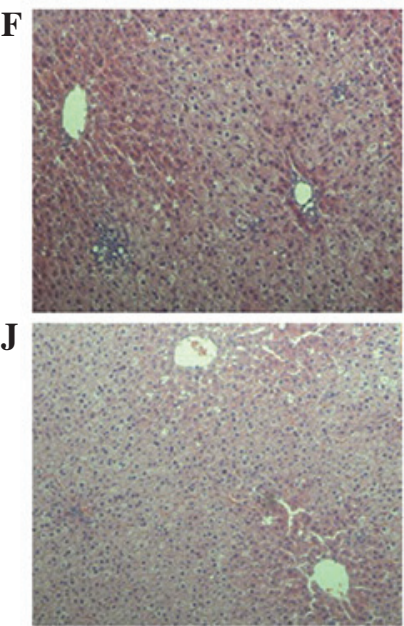
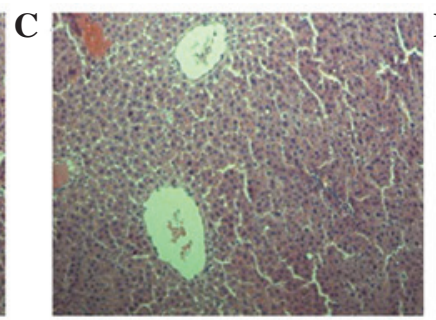

G

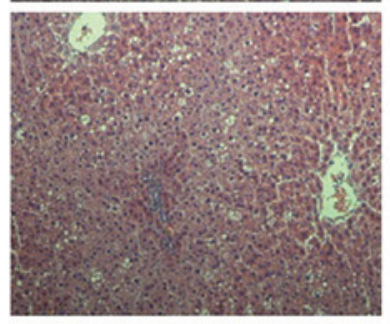

K

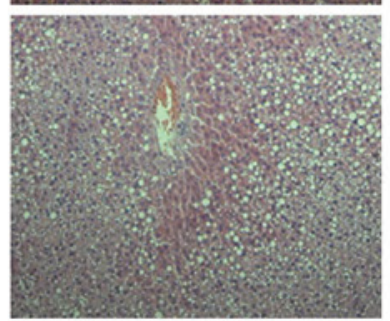

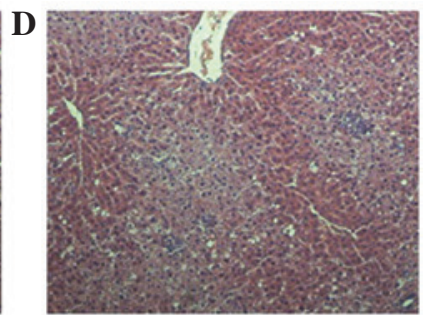

H

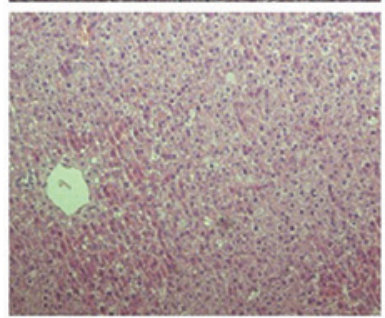

$\mathbf{L}$

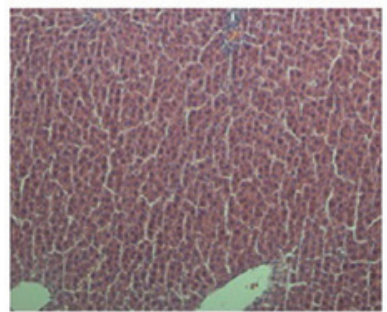

Figure 2. Histopathological changes of liver tissues (hematoxylin-eosin staining; magnification, x100). (A-I) A-I orthogonal experiment groups, respectively; (J) rosiglitazone treatment group; (K) model group; (L) control group.
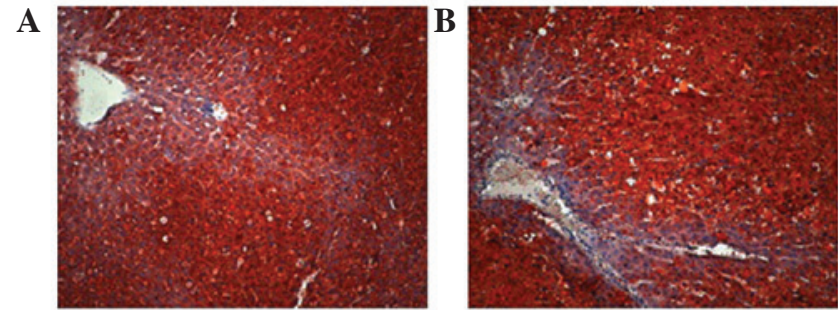

$\mathbf{E}$
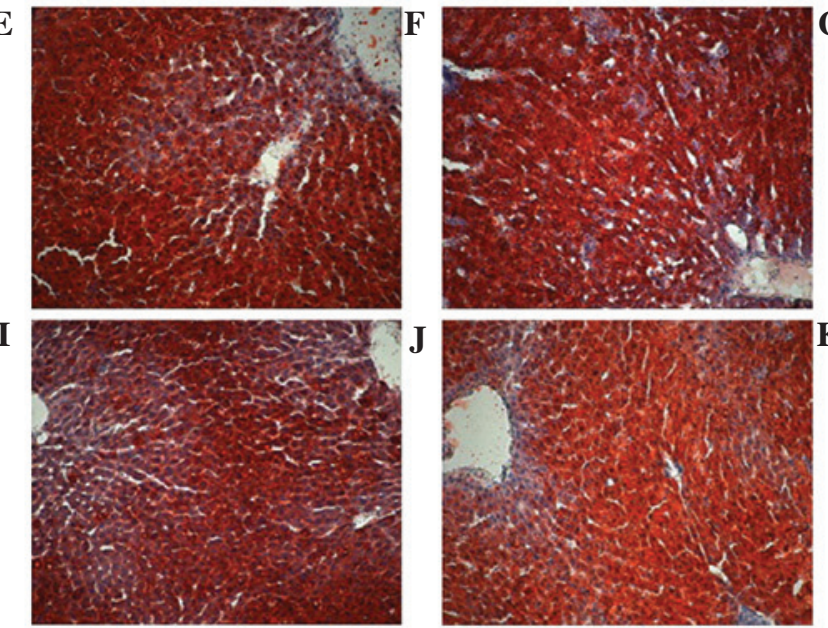
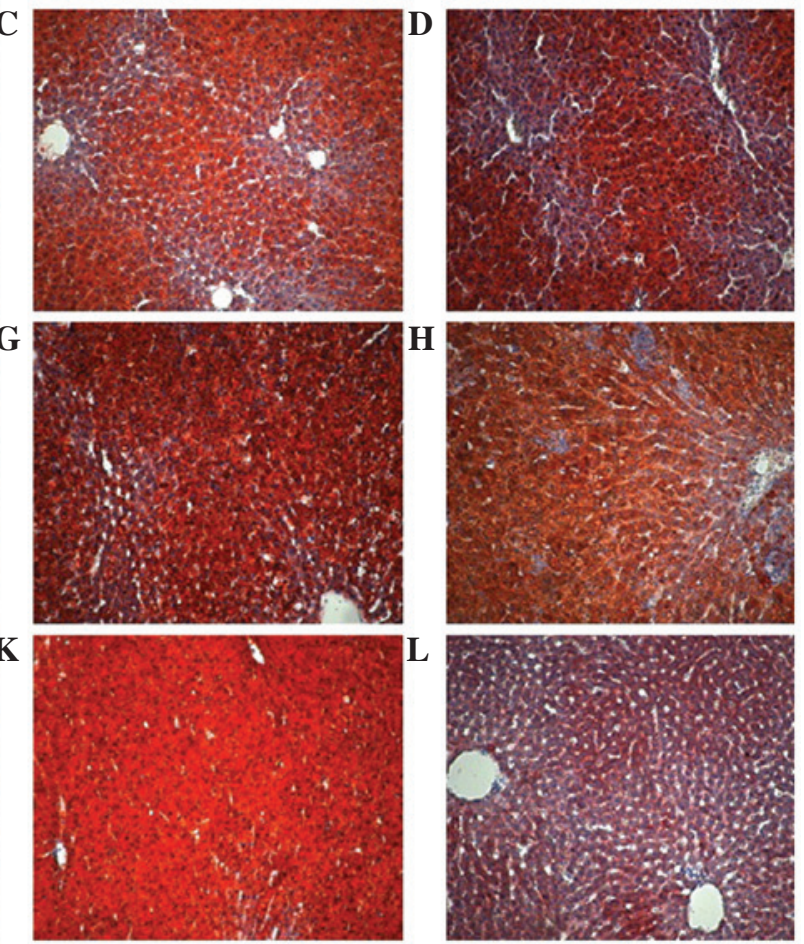

Figure 3. Oil Red O staining of frozen tissue sections (magnification, x100). (A-I) A-I orthogonal experiment groups, respectively; (J) rosiglitazone treatment group; (K) model group; (L) control group.

Plasma ALT and AST levels. The serum levels of ALT and AST were significantly increased $(\mathrm{P}<0.01)$ in the model group, as compared with the control group. Furthermore, in groups F, G, H, I and R the serum levels of AST were significantly decreased $(\mathrm{P}<0.01$, Fig. $5 \mathrm{~A})$, as were the levels of ALT in groups A, B, D, E, I and $\mathrm{R}$ ( $\mathrm{P}<0.01$, Fig. 5B), as compared with the model group.

Effects of puerarin, baicalin, and berberine on the levels of $T N F-\alpha$ and $I L-6$. Compared with the control group, the levels 
A

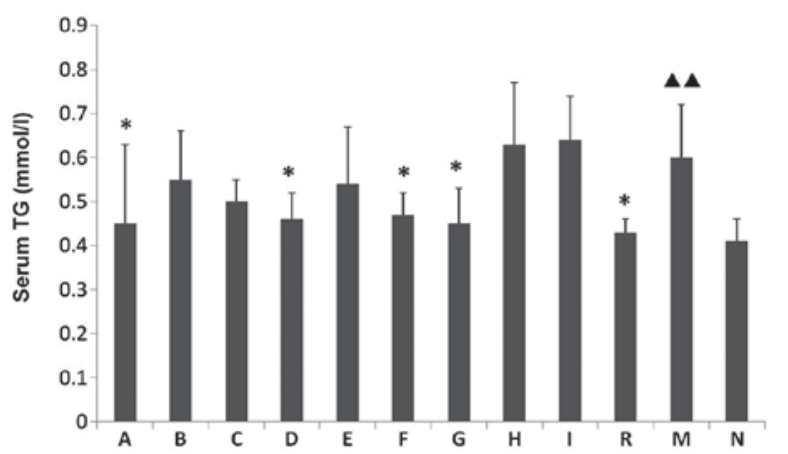

C

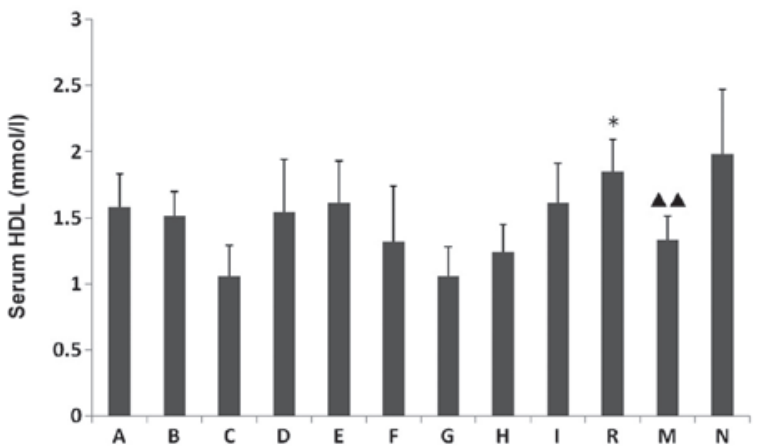

B

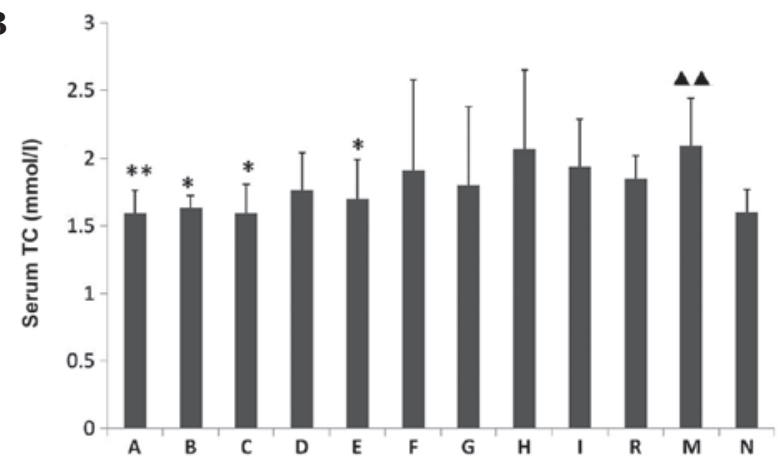

D

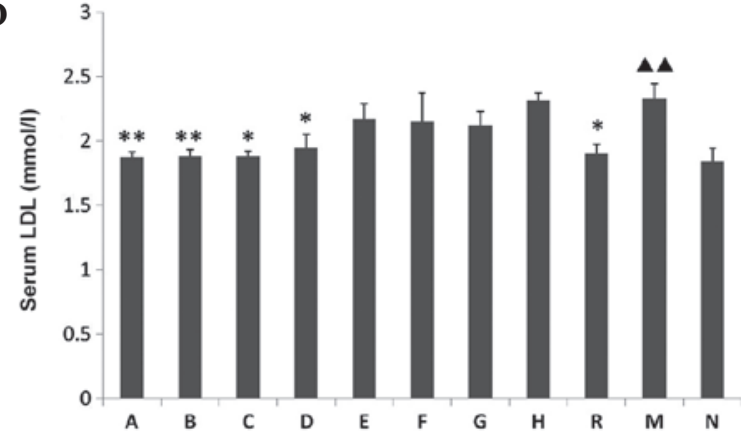

Figure 4. Effects of puerarin, baicalin, and berberine on the serum levels of (A) TG, (B) TC, (C) HDL and (D) LDL in all experimental groups. Data are presented as the mean \pm standard deviation. ${ }^{\mathbf{\Delta}} \mathrm{P}<0.01$, as compared with the normal control group; ${ }^{*} \mathrm{P}<0.05$ and ${ }^{* *} \mathrm{P}<0.01$, as compared with the model group. TG, triglycerides; TC, total cholesterol; HDL, high-density lipoproteins; LDL, low-density lipoproteins; A-I, orthogonal experiment groups A-I; R, rosiglitazone treatment group; $\mathrm{M}$, model group; $\mathrm{N}$, control group.

of TNF- $\alpha$ and IL- 6 in the serum and liver samples of the model group were significantly increased $(\mathrm{P}<0.05)$; whereas, compared with the model group, the serum levels of TNF- $\alpha$ in groups $\mathrm{B}, \mathrm{D}, \mathrm{G}$ and $\mathrm{H}$ were significantly decreased $(\mathrm{P}<0.05$, Fig. 5C), and the serum levels of IL-6 were significantly decreased in groups $\mathrm{A}, \mathrm{B}, \mathrm{D}$ and $\mathrm{F}-\mathrm{H}(\mathrm{P}<0.05$, Fig. 5D). Furthermore, in groups A, D, G, H and I, the levels of TNF- $\alpha$ in the liver were significantly decreased $(\mathrm{P}<0.05$, Fig. 5E), and IL-6 levels in the liver were decreased in groups A, D, F and $\mathrm{H}(\mathrm{P}<0.05$, Fig. $5 \mathrm{~F})$, as compared with the model group.

mRNA expression levels of PPAR- $\gamma$ and IR. In the model group, the mRNA expression levels of PPAR- $\gamma$ and IR were significantly decreased $(\mathrm{P}<0.01)$, as compared with the control group. Furthermore, in groups A, D, G and R the mRNA expression levels of PPAR- $\gamma$ were significantly increased $(\mathrm{P}<0.01$, Fig. $6 \mathrm{~A})$, as were the IR mRNA expression levels in groups A, B, D, F and $\mathrm{R}(\mathrm{P}<0.01$, Fig. 6B), as compared with the model group. However, no significant changes were determined between the rosiglitazone treatment group and the orthogonal experiment groups $(\mathrm{P}>0.05)$.

PPAR- $\gamma$ and IR protein expression levels, as determined by western blotting. Compared with the control group, the protein expression levels of PPAR- $\gamma$ and IR in the model group were significantly decreased $(\mathrm{P}<0.01)$. Furthermore, the PPAR- $\gamma$ protein expression levels in groups $\mathrm{A}, \mathrm{B}, \mathrm{D}, \mathrm{G}, \mathrm{H}$ and $\mathrm{R}$ were significantly increased, as compared with the model group $(\mathrm{P}<0.01$, Fig. $7 \mathrm{~A})$; and in groups $\mathrm{A}-\mathrm{D}, \mathrm{F}$ and $\mathrm{R}$ the protein expression levels of IR were significantly increased $(\mathrm{P}<0.01$,
Fig. 7B). However, no differences were determined between the rosiglitazone treatment and orthogonal experiment groups $(\mathrm{P}>0.05)$.

\section{Discussion}

NAFLD, which has a clinical spectrum ranging from simple fatty liver disease to NASH and cirrhosis (25), is currently the most common type of liver disease (26). Although the etiology of NAFLD has yet to be fully elucidated, the 'two-hit' theory has been accepted as the most popular mechanism (27). The 'first hit' is associated with hepatic TG accumulation and insulin resistance, whereas the 'second hit' involves oxidative stress and the induction of inflammatory cytokines (28). PPAR- $\gamma$ is a sequence-specific and ligand-dependent nuclear transcription factor. With its four isoforms: $\gamma 1, \gamma 2, \gamma 3$ and $\gamma 4$, PPAR- $\gamma$ is associated with the control of lipid storage and the differentiation of adipocytes and macrophages (29). TZDs are high affinity synthetic PPAR- $\gamma$ ligands that can stimulate adipocyte differentiation and improve insulin resistance via PPAR- $\gamma$ activation (30). One TZD in particular, rosiglitazone, has been established as a widely used therapeutic agent in the treatment of insulin resistance. Rosiglitazone has previously been demonstrated to improve sensitivity to insulin as well as transaminases and liver histology (31). In the present study, rosiglitazone was used as a positive pharmacological control, in order to compare the effects of puerarin, baicalin, and berberine on rats with high-fat diet-induced NAFLD. IR was initially identified as a homodimer, with extrinsic disulfide bonds that generate the functional receptor. Each monomer of IR is composed of one $\alpha$ 

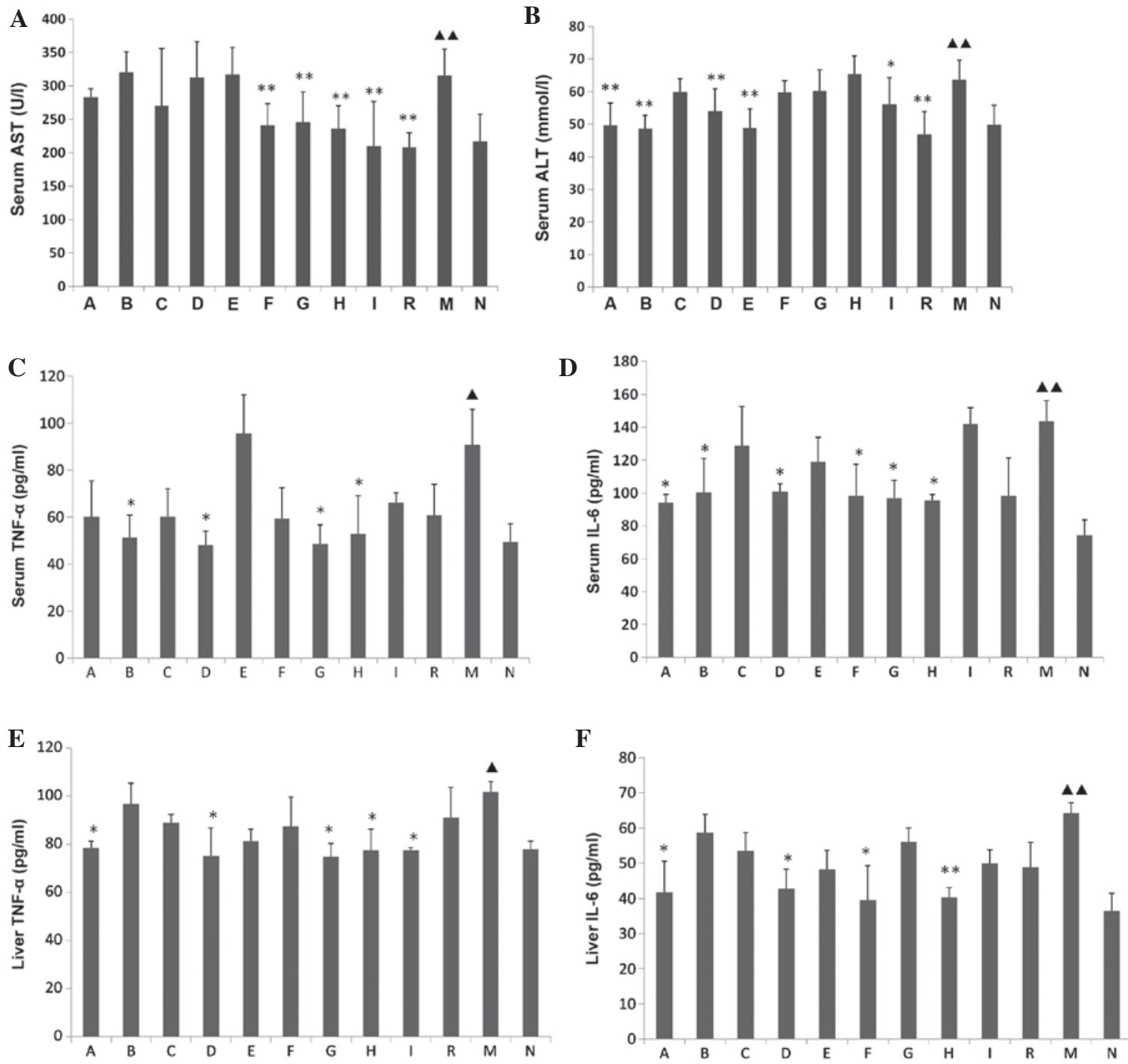

Figure 5. Effects of puerarin, baicalin, and berberine on (A) AST, (B) ALT, (C and E) TNF- $\alpha$ and (D and F) IL-6 levels in all experimental groups. Data are presented as the mean \pm standard deviation. ${ }^{\mathbf{\Delta}} \mathrm{P}<0.05$ and ${ }^{\mathbf{\Delta}} \mathrm{P}<0.01$, vs. the control group; ${ }^{\mathrm{P}} \mathrm{P}<0.05$ and ${ }^{* *} \mathrm{P}<0.01$, vs. the model group. AST, aspartate aminotransferase; ALT, alanine aminotransferase; TNF- $\alpha$, tumor necrosis factor- $\alpha$; IL-6, interleukin-6; A-I, orthogonal experiment groups A-I; R, rosiglitazone treatment group; $\mathrm{M}$, model group; $\mathrm{N}$, control group.
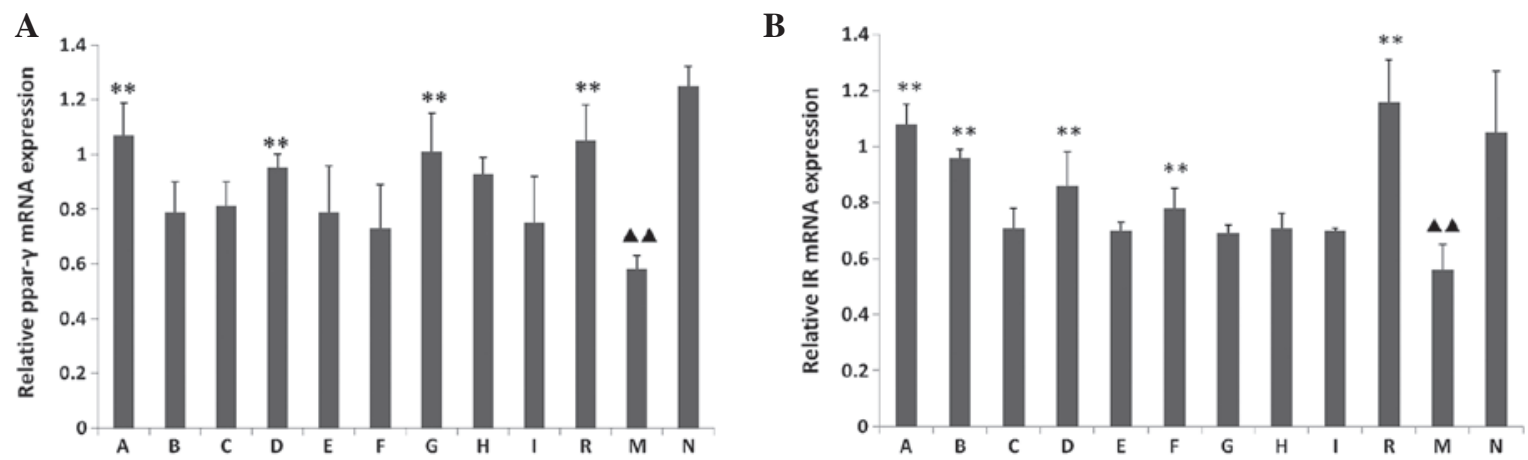

Figure 6. Reverse transcription-quantitative polymerase chain reaction analysis of (A) PPAR- $\gamma$ and (B) IR mRNA expression levels. Data are presented as the mean \pm standard deviation. ${ }^{\mathbf{A} \mathbf{A}} \mathrm{P}<0.01$ vs. the control group; ${ }^{* *} \mathrm{P}<0.01$ vs. the model group. PPAR- $\gamma$, proliferator-activated receptor- $\gamma ;$ IR, insulin receptor ; A-I, orthogonal experiment groups A-I; R, rosiglitazone treatment group; M, model group; N, control group.

and one $\beta$ subunit bridged by an intrinsic disulfide bond $(32,33)$. The lack of IR may directly affect the distribution of insulin in the body, causing insulin resistance and abnormal glucose tolerance. Previous studies have demonstrated that PPAR- $\gamma$ and IR may be important for the 'first hit' stage of NAFLD, since they are associated with insulin resistance $(34,35)$. Increased 

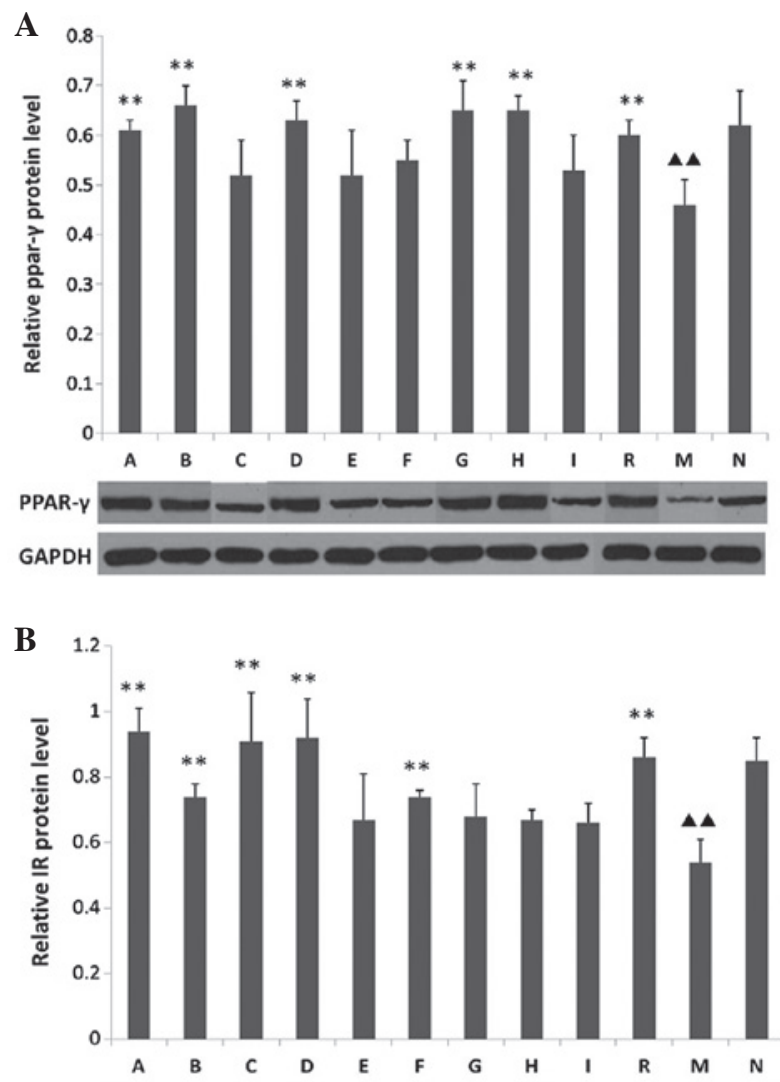

IR

GAPDH

Figure 7. Western blot analysis of (A) PPAR- $\gamma$ and (B) IR protein expression. GAPDH was used as a loading control. Data are presented as the mean \pm standard deviation. ${ }^{\mathbf{\Delta}} \mathrm{P}<0.01$ vs. the control group; ${ }^{* * *} \mathrm{P}<0.01$ vs. the model group. PPAR- $\gamma$, proliferator-activated receptor- $\gamma ;$ IR, insulin receptor ; A-I, orthogonal experiment groups A-I; R, rosiglitazone treatment group; $\mathrm{M}$, model group; $\mathrm{N}$, control group.

PPAR- $\gamma$ also has an anti-inflammatory role as it inhibits the production of various inflammatory cytokines including TNF- $\alpha$, IL-6, IL-1, matrix metalloproteinase-9 and transforming growth factor- $\beta$ (36). Therefore, in the present study TNF- $\alpha$ and IL-6 were selected as representative inflammatory markers involved in the 'second hit' stage.

Since there is still no clear curative treatment for NAFLD, patients may use anti-diabetic, lipid-lowering or anti-hypertensive agents to control NAFLD co-morbidities. Chinese herbs have been traditionally used for treating liver disease worldwide (37), and there are numerous herbal products that are believed to benefit patients with NAFLD, with acceptable levels of safety. For example, previous studies have demonstrated that puerarin, baicalin and berberine, which are three major constituents of the Chinese herbs: Puerariae Radix, Scutellariae Radix and Coptidis Rhizoma, exhibit therapeutic effects on rats with NAFLD (20-22). However, there have been few reports regarding the compatibility of these constituents. The orthogonal experiment is an efficient and economical test, which has the advantage of simultaneously balancing samples and reducing test times, thus ensuring that each test has a strong representation (38). In order to identify improved treatment options for puerarin, baicalin and berberine combination therapy through a small number of experiments that determine the best therapeutic effect, the orthogonal experiment was applied to the overall design, comprehensive comparison and statistical analysis of the present study.

In the present study, rats with high-fat diet-induced NAFLD were employed to evaluate the efficacies of puerarin, baicalin and berberine against NAFLD. The high-fat diet model has been widely used in previous NAFLD studies $(39,40)$. The histological results of the present study observed NAFLD features in the model rats, indicating that an NAFLD model was successfully generated. Furthermore, as expected, the rosiglitazone treatment and orthogonal experiment groups significantly attenuated hepatic steatosis, inflammation and fibrosis in the rats.

The pharmaceutical compositions examined in the present study demonstrated that a combination of puerarin, baicalin and berberine may exert promising lipid-lowering, hepatoprotective and anti-inflammatory activities. The various orthogonal experiment groups had numerous effects on biochemical parameters and pro-inflammatory cytokines. The present study demonstrated that serum levels of TC, LDL and ALT were significantly reduced in the puerarin-dominated groups, whereas serum levels of TNF- $\alpha$ and IL-6 were improved in the baicalin- and berberine-dominated groups. Therefore, the results of the present study suggested that puerarin may preferentially affect lipid metabolism, whereas baicalin and berberine may impact the inflammatory response. Furthermore, PPAR- $\gamma /$ IR protein and mRNA expression levels were also reduced in the model group, thus suggesting that insulin resistance had developed in response to decreased PPAR- $\gamma$ and IR in the NAFLD model rats. Notably, the rosiglitazone treatment group and certain orthogonal experiment groups improved insulin resistance; however, no obvious trends in the various combinations of puerarin, baicalin and berberine were determined. In addition, no differences were demonstrated between the rosiglitazone treatment and orthogonal experiment groups. Therefore, we hypothesize that a combination of puerarin, baicalin and berberine may be used in the early pathological stage ('first hit') of NAFLD to improve insulin resistance via PPAR- $\gamma$ and IR upregulation. Since puerarin treatment is associated with the regulation of lipid metabolism, puerarin may be used in the early stages of simple fatty liver disease; whereas baicalin and berberine may be used in NASH, as they may alleviate the inflammatory response. However, it was not possible to clarify the effects on liver cirrhosis in the present study due to a lack of detection of liver fibrosis.

In conclusion, the combination of puerarin, baicalin and berberine induced favorable therapeutic effects on rats with high-fat diet-induced NAFLD. Furthermore, variably positive effects were demonstrated in the various stages of NAFLD following treatment with numerous proportions of monomer compositions. This suggests that a therapeutic combination of puerarin, baicalin and berberine may regulate lipid metabolism by upregulating the expression of hepatic PPAR- $\gamma$ and IR, leading to improvements in insulin resistance, which may be useful in the prevention and treatment of NAFLD, especially simple fatty liver disease and NASH. Further studies focusing on the effects of combination treatment with puerarin, baicalin and berberine on liver fibrosis are required. 


\section{Acknowledgements}

The present study was supported by the Youth Fund of National Natural Science Foundation of China (grant no. 81503407), Self-selected subject of Beijing University of Chinese Medicine (grant no. 2015-JYB-JSMS125), and the Wang Bao-enLiver Fibrosis Research Fund (grant no. 2013-xjs).

\section{References}

1. Marchesini G and Marzocchi R: Metabolic syndrome and NASH. Clin Liver Dis 11: 105-117, ix 2007.

2. Fabbrini E, Sullivan S and Klein S: Obesity and nonalcoholic fatty liver disease: Biochemical, metabolic and clinical implications. Hepatology 51: 679-689, 2010.

3. Song HY, Zhang L, Pan JL, Yang LL and Ji G: Bioactivity of five components of Chinese herbal formula Jiangzhi granules against hepatocellular steatosis. J Integr Med 11: 262-268, 2013.

4. Stein LL, Dong MH and Loomba R: Insulin sensitizers in nonalcoholic fatty liver disease and steatohepatitis: Current status. Adv Ther 26: 893-907, 2009.

5. Sun L and Lü SZ: Association between non-alcoholic fatty liver disease and coronary artery disease severity. Chin Med J (Engl) 124: 867-872, 2011.

6. Zhao JS, Zhu FS, Liu S, Yang CQ and Chen XM: Pioglitazone ameliorates nonalcoholic steatohepatitis by down-regulating hepatic nuclear factor-kappa B and cyclooxygenases-2 expression in rats. Chin Med J (Engl) 125: 2316-2321, 2012.

7. Zhao CY, Jiang LL, Li L, Deng ZJ, Liang BL and Li JM: Peroxisome proliferator activated receptor-gamma in pathogenesis of experimental fatty liver disease. World J Gastroenterol 10: 1329-1332, 2004.

8. Nan YM, Fu N, Wu WJ, Liang BL, Wang RQ, Zhao SX, Zhao JM and Yu J: Rosiglitazone prevents nutritional fibrosis and steatohepatitis in mice. Scand J Gastroenterol 44: 358-365, 2009.

9. Lutchman G, Modi A, Kleiner DE, Promrat K, Heller T, Ghany M, Borg B, Loomba R, Liang TJ and Premkumar A: The effects of discontinuing pioglitazone in patients with nonalcoholic steatohepatitis. Hepatology 46: 424-429, 2007.

10. Chiu SL and Cline HT: Insulin receptor signaling in the development of neuronal structure and function. Neural Dev 5: 7, 2010.

11. Zhang Q, Xiao XH, Li M, Li WH, Yu M, Zhang HB, Ping F, Wang ZX and Zheng J: Chromium-containing traditional Chinese medicine, Tianmai Xiaoke Tablet improves blood glucose through activating insulin-signaling pathway and inhibiting PTP1B and PCK2 in diabetic rats. J Integr Med 12: $162-170,2014$

12. Samuel VT and Shulman GI: Integrating mechanisms for insulin resistance: Common threads and missing links. Cell 148: 852-871, 2012.

13. Mohammadi A, Gholamhoseinian A and Fallah $\mathrm{H}$ : Zataria multiflora increases insulin sensitivity and PPAR- $\gamma$ gene expression in high fructose fed insulin resistant rats. Iran J Basic Med Sci 17: 263-270, 2014.

14. Rani S and O'Driscoll L: Analysis of changes in phosphorylation of receptor tyrosine kinases: Antibody arrays. Methods Mol Biol 1233: 15-23, 2015.

15. Promrat K, Lutchman G, Uwaifo GI, Freedman RJ, Soza A, Heller T, Doo E, Ghany M, Premkumar A, Park Y, et al: A pilot study of pioglitazone treatment for nonalcoholic steatohepatitis. Hepatology 39: 188-196, 2004.

16. Nesto RW, Bell D, Bonow RO, Fonseca V, Grundy SM, Horton ES, Le Winter M, Porte D, Semenkovich CF, Smith S, et al: Thiazolidinedione use, fluid retention, and congestive heart failure: A consensus statement from the American Heart Association and American Diabetes Association. Diabetes Care 27: 256-263, 2004.

17. Parulkar AA, Pendergrass ML, Granda-Ayala R, Lee TR and Fonseca VA: Nonhypoglycemic effects of thiazolidinediones. Ann Intern Med 134: 61-71, 2001.
18. Wang WJ: Enhancing the treatment of metabolic syndrome with integrative medicine. J Integr Med 11: 153-156, 2013.

19. Yao Z, Zhang L and Ji G: Efficacy of polyphenolic ingredients of Chinese herbs in treating dyslipidemia of metabolic syndromes. J Integr Med 12: 135-146, 2014.

20. Wang Y, Li J, Zhuge L, Su D, Yang M, Tao S and Li J: Comparison between the efficacies of curcumin and puerarin in C57BL/6 mice with steatohepatitis induced by a methionine- and choline-deficient diet. Exp Ther Med 7: 663-668, 2014.

21. Guo HX, Liu DH, Ma Y, Liu JF, Wang Y, Du ZY, Wang X, Shen JK and Peng HL: Long-term baicalin administration ameliorates metabolic disorders and hepatic steatosis in rats given a high-fat diet. Acta Pharmacol Sin 30: 1505-1512, 2009.

22. Chang X, Yan H, Fei J, Jiang M, Zhu H, Lu D and Gao X: Berberine reduces methylation of the MTTP promoter and alleviates fatty liver induced by a high-fat diet in rats. J Lipid Res 51: 2504-2515, 2010.

23. Cui W, Li X, Zhou S and Weng J: Investigation on process parameters of electrospinning system through orthogonal experimental design. J Appl Polym Sci 103, 3105-3112, 2007.

24. Khan F, Choong WL, Du Q and Jovanovi'c A: Real-time RT-PCR $\mathrm{Ct}$ values for blood GAPDH correlate with measures of vascular endothelial function in humans. Clin Transl Sci 6: 481-484, 2013.

25. Matteoni CA, Younossi ZM, Gramlich T, Boparai N, Liu YC and McCullough AJ: Nonalcoholic fatty liver disease: A spectrum of clinical and pathological severity. Gastroenterology 116: 1413-1419, 1999.

26. Adams LA and Angulo P: Treatment of non-alcoholic fatty liver disease. Postgrad Med J 82: 315-322, 2006.

27. Day CP and James OF: Steatohepatitis: A tale of two 'hits'? Gastroenterology 114: 842-845, 1998.

28. Postic C and Girard J: Contribution of de novo fatty acid synthesis to hepatic steatosis and insulin resistance: Lessons from genetically engineered mice. J Clin Invest 118: 829-838, 2008

29. Law RE, Goetze S, Xi XP, Jackson S, Kawano Y, Demer L, Fishbein MC, Meehan WP and Hsueh WA: Expression and function of PPARgamma in rat and human vascular smooth muscle cells. Circulation 101: 1311-1318, 2000.

30. Harrison SA: Thiazolidinedione therapy for nonalcoholic steatohepatitis: Go, stop, or proceed with caution? Hepatology 51: 366-369, 2010.

31. Nan YM, Fu N, Wu WJ, Liang BL, Wang RQ, Zhao SX, Zhao JM and $\mathrm{Yu}$ J: Rosiglitazone prevents nutritional fibrosis and steatohepatitis in mice. Scand J Gastroenterol 44: 358-365, 2009.

32. Cheatham B and Kahn CR: Cysteine 647 in the insulin receptor is required for normal covalent interaction between alpha- and beta-subunits and signal transduction. J Biol Chem 267: 7108-7115, 1992.

33. Seino S and Bell GI: Alternative splicing of human insulin receptor messenger RNA. Biochem Biophys Res Commun 159: 312-316, 1989.

34. El-Bassossy HM, Abo-Warda SM and Fahmy A: Chrysin and luteolin alleviate vascular complications associated with insulin resistance mainly through PPAR- $\gamma$ activation. Am J Chin Med 42: 1153-1167, 2014.

35. Shamsi BH, Ma C, Naqvi S and Xiao Y: Effects of pioglitazone mediated activation of PPAR- $\gamma$ on CIDEC and obesity related changes in mice. PLoS One 9: e106992, 2014.

36. Wang RC and Jiang DM: PPAR- $\gamma$ agonist pioglitazone affects rat gouty arthritis by regulating cytokines. Genet Mol Res 13: 6577-6781, 2014.

37. Seeff LB, Lindsay KL, Bacon BR, Kresina TF and Hoofnagle JH: Complementary and alternative medicine in chronic liver disease. Hepatology 34: 595-603, 2001.

38. Liu RJ, Zhang YW, Wen CW and Tang J: Study on the design and analysis methods of orthogonal experiment. Exp Technol and Manage 27: 52-55, 2010.

39. Silva RN, Bueno PG, Avó LR, Nonaka KO, Selistre-Araújo HS and Leal AM: Effect of physical training on liver expression of activin $\mathrm{A}$ and follistatin in a nonalcoholic fatty liver disease model in rats. Braz J Med Biol Res 47: 746-752, 2014.

40. Kucera O and Cervinkova Z: Experimental models of nonalcoholic fatty liver disease in rats. World J Gastroenterol 20: 8364-8376, 2014. 